\title{
IMAGING PLATES \\ IN SYNCHROTRON AND CONVENTIONAL X-RAY CRYSTALLOGRAPHIC DATA COLLECTION
}

\author{
Z. DaUter and K.S. Wilson \\ European Molecular Biology Laboratory, Outstation Hamburg \\ c/o DESY, Notkestrasse 85, 22603 Hamburg, Germany
}

\begin{abstract}
Application of two-dimensional detectors based on imaging plates for $\mathrm{X}$-ray crystallographic data collection is discussed. The properties of imaging plates are somewhat different from those of other available X-ray detectors, like film, multiwire ionization chambers, TV tubes or scintillation counters. Imaging plate scanners are well suited for laboratory $\mathrm{X}$-ray sources as well as for strong synchrotron radiation. Originally developed for macromolecular crystallography, they can be used successfully to collect data on crystals of small or medium size molccules.
\end{abstract}

PACS numbers: $07.85 .+n, 29.40 . G x$

\section{Introduction}

Data collection is one of the most vital stages in structural crystallography. Once suitable crystals have been grown, it is important to collect as good quality data as possible. Later stages of crystallographic analysis use measured reflection intensities for calculation of initial phases, electron density maps and least-squares refinement. The accuracy of the final structural atomic model depends crucially on the precision of the intensities measured during data collection. Some techniques, e.g. anomalous scaltering phasing or charge density calculations, require particularly accurately estimated intensities.

Advances in computing make it possible to attack effectively structures of very large molecules, protein complexes or viruses, consisting of many thousands of atoms, where often more than a million reflections have to be measured and processed. Ilere the speed of the detector plays an important role. The larger the structure, the weaker are the diffracted reflection intensities on average and hence the need for a strong radiation source and a sensitive detector. In addition automatization of the data collection and reduction process is desirable.

Several detectors are a vailable for collecting X-ray diffraction data. Single counter diffractometers employ scintillation counters to measure the intensity of individual reflections one after another scanning through their profile one-dimensionally. They usually have a four-circle goniostat to orient appropriately the crystal 
and set the counter $2 \theta$ angle in order to select the desired reflection. The movements of all arcs are controlled by computer. Although four-circle diffractometers provide accurately measured intensities, they are rather slow devices, used mostly in small molecule crystallography. A diffractometer can deliver several hundred intensities per day.

Two-dimensional detectors have a large active window consisting of individual pixels and can record many reflections simultaneously. Usually the rotation method of data collection is employed on these devices, where a crystal is smoothly rotated around only one axis during exposure to the $\mathrm{X}$-ray beam. As a result whole two-dimensional reflection profiles are recorded on the detector together with a large background area. The range of rotation per image is limited by the number of reflections collected; overlap of the profiles should be avoided. For protein crystals this limit is about $1^{\circ}$, for small structures several degrees. Too wide rotation ranges result in an increase in background and a decrease in signal to noise ratio. In practice three approaches are used, depending on the relation of rotation range $(\Delta \phi)$ to the effective rocking curve $(\eta)$ of the crystal, which takes up its mosaicity and beam divergence:
Fine slicing
Intermediate
$\Delta \phi<\eta$,
$\Delta \phi \approx \eta$
Wide rotation
$\Delta \phi>\eta$.

In the wide rotation mode most of reflections have their whole profile recorded on one image (fully recorded reflections). In the fine slicing mode every reflection intensity is spread over several consecutive images and the total intensity has to be summed from the three-dimensional profile. In the intermediate mode, where the rotation range is comparable with the rocking curve, some reflections are fully recorded and some partially recorded on two or more successive images.

On two-dimensional detectors intensities have to be integrated from the individual pixels containing the complete reflection profile taking into account the surrounding background. Before integration, images have to be indexed, i.e. the crystal unit cell and orientation matrix has to be calculated so that for every reflection it is possible to predict its position on the detector. There are several programs available which can be employed to integrate and reduce the intensities from raw two-dimensional images.

In wide rotation mode, integration is usually performed after completing data collection and complete images are stored on computer disk. For photographic film it is necessary to scan all films on an optical scanner. In fine slicing mode there is a large number of images and often they are processed on-line without the need to store them all.

In EMBL Ilamburg one of the first automatic imaging plate scanners was constructed in 1988 by IIendrix and Lentfer. Now all three protein crystallography synchrotron beam lines are equipped with imaging plates and one scanner is mounted on the sealed tube source. In recent years several hundreds of data sets have been collected on protein crystals mainly using synchrotron radiation and, to a lesser extent, a Mo or $\mathrm{Cu}$ sealed tube. Several data sets were also collected on crystals of small or medium sized organic compounds using both types of radiation sources. Some results and conclusions from the experience of collecting X-ray 
diffraction data at EMBL are presented in this paper, after firstly explaining in more detail how the imaging plates work.

\section{Imaging plates as $\mathrm{X}$-ray detectors}

Imaging plates have been developed by Fuji and Kodak for use in medical $\mathrm{X}$-ray applications. In the late 1980's several groups independently showed their potential as detectors in X-ray crystallography [1].

An imaging plate has the form of a flexible plastic sheet with a layer of active material on one side. The photostimulatable phosphor contains barium halide doped with europium ( $\mathrm{BarBr}: \mathrm{Eu}^{2+}$ ). The $\mathrm{X}$-ray quanta are stored in the form of metastable excited Eu states, so-called colour centres. After exposure to $\mathrm{X}$-rays red laser light is used to deactivate colour centres with emission of blue light as photostimulated luminescence. The intensity of the blue light is proportional to the amount of stored $\mathrm{X}$-rays. It can be measured by a photomultiplier system pixel by pixel and written to computer disk in digitised form. Images should be corrected according to the response of individual pixels. The laser wipes out most of the colour centres, the rest are erased by a few seconds flash of a white lamp. The plate is then ready for the next exposure. The whole cycle is illustrated in Fig. 1.

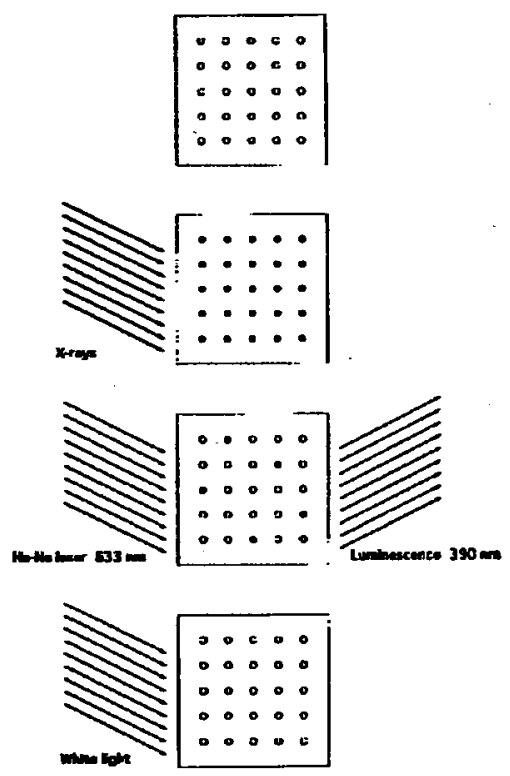

Fig. 1. Imaging plate work cycle consisting of exposure to X-rays, read-out by He-Ne laser with photoluminescence of the blue light and erasure of the residual signal by the white lamp. 
In principle imaging plates can be treated as a kind of re-usable film. The first prototype scanner in IIamburg delivered several thousands of images from its single plate without any visible sign of deterioration of data quality. IIowever, in comparison with film imaging plates have several superior properties.

The imaging plate exposure/read-out/erasure cycle can be completely automated in a single device, the scanner. Imaging plates do not require any chemical processing, which for film is very difficult to control and may lead to non-uniform response within an individual film or between films.

Imaging plates are exposed directly by $\mathbf{X}$-rays scattered from the crystal with subsequent pixel-by-pixel read-out and produce images which are not distorted geometrically, especially by on-line devices where exposure and scanning takes place automatically within the same scanner. Some other two-dimensional detectors, e.g. multiwire gas chamber devices, require additional distortion calibration and correction.

Unlike film, imaging plates have high sensitivity over a wide range of wavelengths. It diminishes somewhat at short wavelengths, but rises again below the barium absorption edge at $0.33 \AA$. The use of short wavelength radiation diminishes absorption effects and reduces the radiation damage of sensitive crystals.

In contrast to other two-dimensional detectors (except charge coupled devices) imaging plates have a very high usable dynamic range of at least $1: 10^{6}$ in which the response is linear. In practice the maximum stored pixel intensity of the imaging plates is limited by the number of bits transferred by the electronics of the read-out system. For strongly diffracting crystals it may be necessary to record a second data set with shorter exposure times, to cover adequately the overloads, i.e. intensities too high to be measured with longer exposures.

The intrinsic spatial resolution of the imaging plate is high, with FWIIM of the point spread function about $150 \mu \mathrm{m}$. In practice it depends on the selected pixel size and the optics of the read-out system. Recently a new kind of bluc-coloured imaging plate was introduced by Fuji, with even higher spatial resolution [2]. The smaller the pixel size, the longer the scanning time. However, imaging plates may have large dimensions. The size of the plates used at the synchrotron beam lines at the Photon Factory ranges up to $600 \mathrm{~mm}$. The possibility of using large size active recording area of the detector at long distance from the crystal is important for reducing the signal to noise ratio, especially with a helium filled path between crystal and detector. The background is then spread out over a larger area with the width of reflections depending on the beam collimation and crystal size.

Another advantage of imaging plates is their negligible intrinsic noise, in contrast to serious effect of chemical fog on photographic film and "dark current" of TV detectors. The effect of fading out of the latent image stored on the plate is not significant if the scan takes place within minutes after exposure.

The draw-back of imaging plates in comparison with TV or ionisation chamber detectors is their significant scanning time. The time necessary to scan the image pixel by pixel by the laser is a few minutes. For on-line scanners that is a dead-time between exposures. Off-linc scanners may require several interchangeable plates. The dead-time is serious only at intense synchrotron beam lines where exposures take seconds, but is less important for sealed tube or rotating anode 
sources where exposures are longer than scanning time. Imaging plates are not well suited for fine slicing mode of data collection at synchrotron radiation sources.

The properties of different detectors are summarised in Table I.

TABLE I

Comparison of the properties of different X-ray detectors.

\begin{tabular}{l|c|c|c|c|c|c}
\hline \hline & Difo & Film & II & MWPC & TV & CCD \\
\hline Dynamic range & II & L & II & L & M & II \\
Sensitivity & II & L & II & II & M & II \\
Spatial resolution & L & I & II & M & M & M \\
Speed & L & L & M & M & II & II \\
\hline
\end{tabular}

Diflo - 4-circle diffractometer, Film - photographic film, IP - imaging plate, MWPC - multiwire proportional chambers, TV - television detectors, CCD - charge coupled devices; II - high, M - medium, L - low.

Several imaging plate scanners are commercially available. The most popular ones are manufactured by MAR Research, Rigaku and MAC Science. They differ in some technological details, but all can produce high quality data.

The MAR scanner las a single circular imaging plate of 300 or $180 \mathrm{~mm}$ diameter with $150 \mu \mathrm{m}$ pixels. After exposure the plate rotates while the laser head moves along the radius of the disc during the scan, which takes 4 minutes for the big plate and below 2 minutes for the smaller one. This set-up ensures high mechanical stability. The resulting set of spiral pixels has to be transformed and interpolated to a Cartesian system for subsequent processing. The scanner is controlled by a computer work-station. All EMIBL beam lines are equipped with MAR scanners or their prototype, developed in-house. In fact all synchrotron facilities in Europe, USA and in Japan currently have different kinds of imaging plates in use.

The Rigaku R-axis scanner [3] has two square plates which can be interchanged between the exposing and scanning positions. The plate is scanned line after line with a grid of $2000 \times 2000$ pixels. The scanning of one plate takes 8 minutes. MAC Science offers several imaging plate scanners having either a flat or cylindrically bent plate optionally coupled with Weissenberg translation.

A unique imaging plate system is a set-up at the Photon Factory synchrotron facility in Tsukuba, Japan [4]. The off-line system has several exchangeable cylindrical cassettes for imaging plates of different dimensions, ranging up to more than half a meter. It is possible to couple the rotation of the crystal with the translation of the plate along the rotation axis, employing classic Weissenberg geometry. This allows the use of extremely wide rotation ranges, up to $20^{\circ}$ per image, without overlap of spots. To diminish background resulting from air scatter the cassettes are filled by helium.

Several programs are available for processing raw images. They are usually modified versions of the soltware developed originally for film processing (MOSFLM [5], DENZO [6]) or ionisation chamber detectors (XDS [7]). The programs are 
rather elaborate, but also robust and automatic. The data processing algorithms consist in bricf of the following steps:

- estimation of the crystal unit cell and orientation matrix (autoindexing),

- prediction of all reflections and their positions on all images,

- least-squares refinement of all relevant parameters (cell dimensions, orientation matrix, mosaicity, distortion parameters etc.) based on differences between observed and predicted spot positions,

- evaluation of reflection intensities by integrating over all contributing pixels taking into account the surrounding background,

- application of the necessary corrections (Lorentz, polarization, oblique incidence),

- scaling of all images on the basis of intensities of the symmetry equivalent reflections,

- summing the partially recorded intensities on consecutive images,

- merging all symmetry equivalent reflections to produce the unique data.

Although most of these steps can be performed automatically, care should be taken to avoid any mis-interpretation. The programs provide thorough diagnostic output and all necessary parametcrs can be refined or adjusted. The choice of the data processing program remains a matter of user preference. The final data can be obtained a few hours after the experiment.

The evaluation of standard deviations of measured intensities deserves comment. A diffractometer provides standard deviations calculated directly from counting statistics, because the scintillation counter measures the individual $\mathrm{X}$-ray quanta scattered by the crystal. Two-dimensional detectors, like imaging plates; measure the $X$-ray quanta indirectly and as a result the final level of standard deviations may not be the same as that of the integrated intensities. The standard deviations should be therefore adjusted to the statistically expected level during data merging. The data processing programs provide this option.

As stated earlier, it is worth taking care and eflort to collect and process diffraction data carefully, to avoid any future complications resulting from doubtful or non-optimal data quality.

In protein crystallography inaging plate scanners are currently the most popular detectors, both in home and synchrotron laboratories. In future they will probably give way to charge coupled devices but at present the CCD technology is not yet sufficiently advanced. Only for special purposes is film still used, e.g. in Laue diffraction experiments, where extremely high spatial resolution is required.

\section{Selected results}

All examples presented here come from the experience of using imaging plate scanners at EMBL in Ilamburg. The crystals involved range from the very small structure of urotropin to a large unit cell of a virus.

The ability of the imaging plate to give data of extremely high quality can be illustrated by the test performed on a crystal of potassium monotartrate, 
TABLE II

Summary of data collection and refinement of KAMTRA.

\begin{tabular}{|c|c|c|c|}
\hline \multirow{3}{*}{$\begin{array}{l}\text { Cell dimensions } \\
\text { Temperature [K] } \\
\text { No. of images }\end{array}$} & \multicolumn{3}{|c|}{$\begin{array}{c}a=7.8, b=10.7, c=7.6 \AA \\
P 2_{1} 2_{1} 2_{1} Z=4\end{array}$} \\
\hline & 293 & 120 & 85 \\
\hline & $19 \quad 10$ & $20 \quad 11$ & 135 \\
\hline$\Delta \phi\left[^{0}\right]$ & 10 & 10 & 10 \\
\hline Distance [mm] & $\begin{array}{ll}73 & 130\end{array}$ & $85 \quad 140$ & $85 \quad 140$ \\
\hline Resolution $[\AA]$ & $\begin{array}{ll}0.85 & 1.2 \\
\end{array}$ & $0.9 \quad 1.3$ & $0.9 \quad 1.3$ \\
\hline Measurements & 3222 & 2678 & 15936 \\
\hline Unique (Friedels merged) & 614 & 528 & 536 \\
\hline$R(I)$ symm & 0.026 & 0.024 & 0.023 \\
\hline$R(I) \sigma$ & 0.040 & 0.040 & 0.016 \\
\hline Observables & 1009 & 858 & 872 \\
\hline Parameters & 121 & 121 & 121 \\
\hline $\mathrm{w} R 2$ & 0.056 & 0.044 & 0.041 \\
\hline$>4 \sigma(F)$ & 993 & 850 & 870 \\
\hline$R 1$ & 0.025 & 0.015 & 0.014 \\
\hline GooF & 0.86 & 0.73 & 0.89 \\
\hline Flack $x$ & -0.02 & 0.005 & 0.01 \\
\hline
\end{tabular}

$\mathrm{KHIC}_{4} \mathrm{H}_{4} \mathrm{O}_{6}$ (KAMTRA). The crystal was ground to spherical shape, mounted on the top of a beryllium rod and enclosed within a low-absorption glass capillary. Three experiments were carried out at different temperatures, 293, 120 and $85 \mathrm{~K}$ on a small MAR scanner with Mo $K_{\alpha}$ radiation, firstly collecting the high resolution, longer exposure images and subsequently the low resolution, short exposure data. Table II summarises the results of data collection and structure refinement against these data on $\mathrm{F}^{2}$ with Friedel mates separated, using the program SIIELXL93. "Strong" and "weak" data sets were collected separately and merged together after scaling. This crystal was used previously as a standard test in the laboratory of P. Luger at the Free University, Berlin [8]. Each data set required about 12 hours to collect, except at $85 \mathrm{~K}$, where very highly redundant data were collected. In addition to very low final discrepancy factors, the value of the Flack $x$ parameter discriminating the two enantiomorphs refined very close to the expected value of 0 $( \pm 0.04)$ confirming the high accuracy of the measured anomalous signal. The value of the imaginary anomalous correction $\delta f^{\prime \prime}$ for potassium at $0.71 \AA$ wavelength is about 0.25 of an electron.

One of the smallest ever crystals, in terms of its cell dimensions and number of atoms, on which $\mathrm{X}$-ray data were collected by the rotation method, is urotropin or 1,3,5,7-tetraazaadamantane [9]. The molecule of urotropin, $\mathrm{C}_{6} \mathrm{II}_{12} \mathrm{~N}_{4}$, is highly symmetric, $\overline{4} 3 \mathrm{~m}$, and lies at a special position of the cubic space group $I \overline{4} 3 \mathrm{~m}$ with cell dimension $7.03 \AA$. There are only three independent atoms in the structure 
and all three lie on special positions. There are 15 parameters to refine including anisotropic displacement parameters of hydrogen and overall scale.

The diffraction data were collected on a $180 \mathrm{~mm}$ MAR scanner and Mo sealed tube and also, for comparison, on a four-circle diffractometer with $\mathrm{Cu}$ radiation. Both set-ups provide the same ligh resolution limit of $0.8 \AA$. On the diffractometer the standard procedure was employed to collect a highly redundant set of data, about 2500 intensities, which reduced to 52 unique reflections. On the imaging plate about the same number of individual intensities wcre measured at two distances with different exposure times and rotation ranges $10^{\circ}$ and $15^{\circ}$. The two sets of intensities appeared to be equivalent in quality and led to refined structural models of comparable, high accuracy with an $R$ factor of $3 \%$. The main difference in these two experiments was the time required for data collection. The total crystal exposure time on the diffractometer was 39 hours whereas the imaging plate scanner required 7 hours. Obviously for larger structures the time required for data collection favours imaging plate more strongly.

The imaging plate as two-dimensional detector for small structures provides the opportunity to observe diffraction effects betwcen Bragg reflections. Splitting of the crystal, satellite reflections, thermal diffuse scattering etc. can be easily seen during data collection. These effects are hard to judge on the diffractometer.

About thirty data sets were collected using imaging plate scanner and Mo sealed tube at EMBL on organic crystals, their structures solved and refined successfully. The usefulness of the imaging plate for recording X-ray diffraction data on small structure crystals does not need to be proved further.

The full potential of imaging plates was shown in macromolecular applications. Introduction of imaging plate scanners had a very important influence especially at synchrotron sites. Previously film was used routinely because of its high spatial resolution. Because of the limitation in dynamic range of photographic film, multifilm packs were used, which involved extensive, very tiresome scanning of films on optical scanncrs before data processing. On imaging plates it is possible to process the first images and propcrly judge data quality at the beginning of data collection. With films the wavelength of choice was about $1.5 \AA$, whereas with imaging plates wa velengths below $1 \AA$ are routincly used which is much less damaging for sensitive protein crystals and largely alleviates absorption effects.

Data collection on crystals of Carnation Mottle Virus [10] illustrates very well the advantage of using short wavelengths. Some data had previously been collected on these crystals on film with $1.488 \AA$ radiation and under these conditions crystals could withstand only one or two exposures. With a wavelength of $1 \AA$ and imaging plate scanner as detector it was possible to obtain about 30 exposures from one crystal. $91 \%$ complete (outstanding for virus work) data to $3.2 \AA$ resolution were collected from thrce crystals and a fourth one covering the low resolution $5.5 \AA$ region during a $24 \mathrm{~h}$ synchrotron session. The virus crystallises in cubic space group $I 23$ with a cell dimension $a=382 \AA$. A bout 1.5 million measurements were merged to 140000 unique reflections with $R(I)=8.2 \%$. The structure was easily solved by molecular replacement and refined with 5 -fold non-crystallographic symmetry restraints to an $R$ factor of $18 \%$. The model comprises 7479 protein atoms [10]. 
The structure determination of dUTPase [11] shows the importance of accurate measurements of the anomalous scattering signal from heavy atom at optimised wavelength. The enzyme crystallises in $R 3$ space group with $a=86.6$, $c=62.3 \AA$. Native data were collected to a resolution of $1.9 \AA$. Data for two heavy atom derivatives, IIg to $2.0 \AA$ at a wavelength of $0.995 \AA$ (IIg $L_{\text {III }}$ absorption edge is $1.009 \AA$ ) and $\mathrm{Pt}$ to $2.1 \AA$ at $1.050 \AA$ wa velength ( $\mathrm{Pt} L_{\mathrm{III}}$ edge is $1.07 \AA$ ) were collected on an imaging plate scanner at the X31 ENBL beam line, which has a double channel-cut monochromator and is particularly suited for anoma-
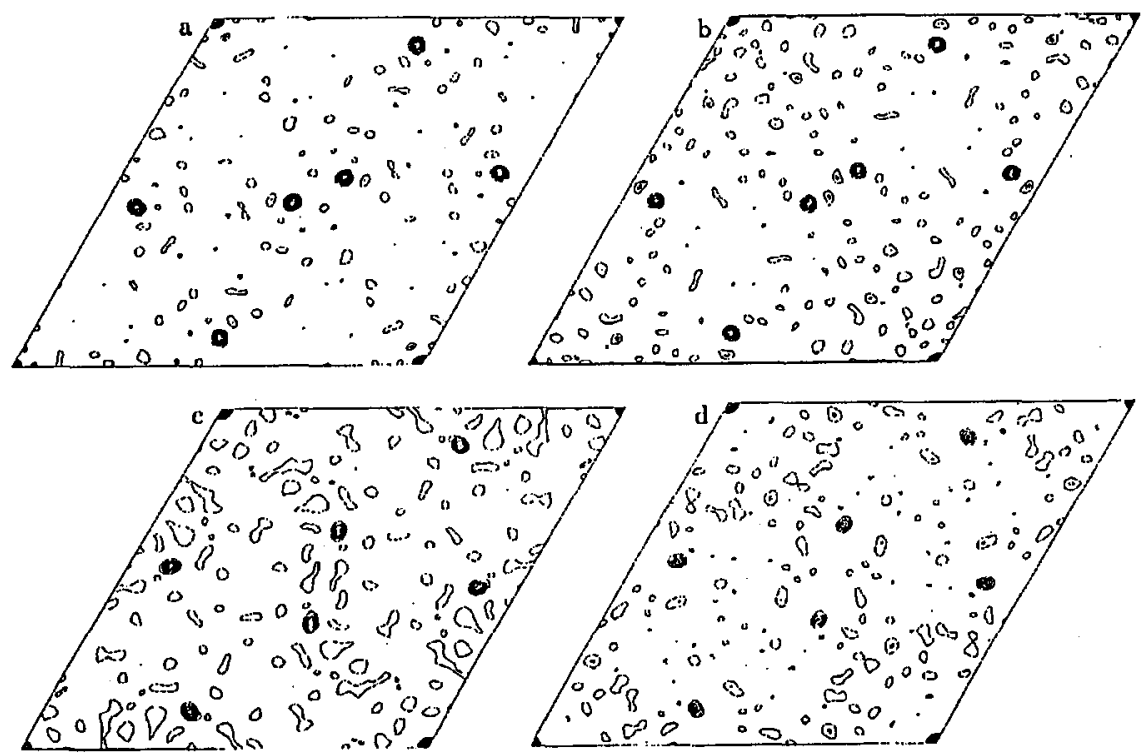

Fig. 2. IIarker sections $w=0$ of the Palterson syntheses for heavy atom derivatives of dUTPase, based on (a) IIg isomorphous differences, (b) IIg anomalous differences, (c) $\mathrm{Pt}$ isomorphous differences, (d) $\mathrm{P} t$ anomalous differences.

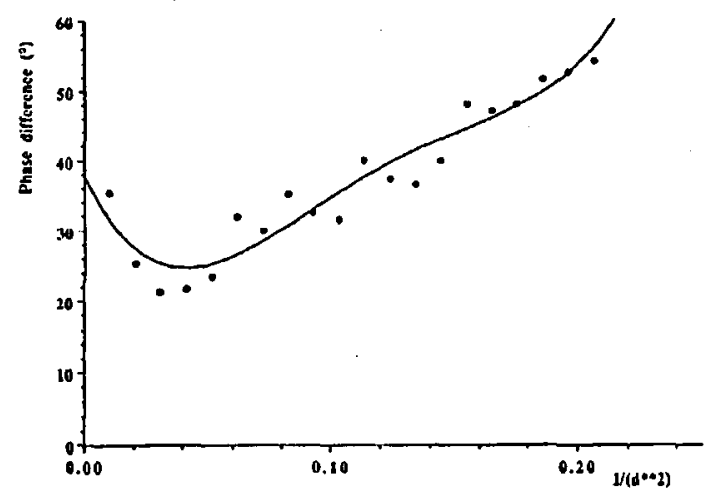

Fig. 3. Average differences between initial and final phases as a function of resolution $d$. 
lous diffraction work. Figure 2 shows the $w=0$ IIarker sections of Patterson syntheses based on isomorphous and anomalous differences for both derivatives. The peaks corresponding to single sites are clearly seen for both derivatives, for $\mathbf{P t}$ site the occupancy is only partial. The initial phases obtained on the basis of the two derivatives produced a very clear electron density map, easily interpretable in terms of the protein chain. Figure 3 shows the differences between the initial MIR and final phases calculated from the refined model as a function of resolution $d$. The average phase difference was $40^{\circ}$ to $2.1 \AA$, but phase differences are less than $30^{\circ}$ at lower resolution.

\section{References}

[1] Y. Amemiya, J. Miyahara, Nature 336, 89 (1988).

[2] M. Sato, Y. Katsube, K. IIayashi, J. Appl. Cryst. 26, 733 (1993).

[3] M. Sato, M. Yamamoto, K. Inada, Y. Kalsube, N. Tanaka, T. IIigashi, J. Appl. Cryst. 25, 348 (1992).

[4] N. Sakabe, Nucl. Instrum. Melhods Phys. Res. A 303, 448 (1991).

[5] A.G.W. Leslie, P. Brick, A.J. Wonacott, Collaborative Computing Project No. 4 Newsletter 18, 33 (1986).

[6] Z. Otwinowski, DENZO: An Oscillation Data Processing Program for Macromolecular Crystallography, Yale University, New IIaven 1993.

[7] W. Kabscl, J. Appl. Cryst. 21, 916 (1988).

[8] P. Luger, Modern X-Ray Analysis on Single Crystals, de Cruyter, Berlin 1980.

[9] J. Grochowski, P. Serda, K.S. Wilson, Z. Dauter, J. Appl. Cryst. 27, 1994, in press,

[10] E.Y. Morgunova, Z. Dauter, E. Fry, D.I. Stuart, V.Y. Stelmashchuk, A.M. Mikhailov, K.S. Wilson, B.K. Vainshtein, Federation of European Biochemical Societies Newsletler 338, 267 (1991).

[11] E.S. Cedergren-Zeppezauer, G. Larsson, P.O. Nyman, Z. Dauter, K.S. Wilson, Nature 355, 740 (1992). 